\title{
Percepções e Práticas Alimentares de Estudantes de Gastronomia de uma
} Universidade Pública Brasileira

\author{
Perceptions and Food Practices of Gastronomy Students at a Brazilian Public University \\ Percepciones y Prácticas Alimentarias de Estudiantes de Gastronomía de una Universidad Pública
}

Brasileña

Recebido: 24/02/2021 | Revisado: 04/03/2021 | Aceito: 10/03/2021 | Publicado: 17/03/2021

Samuel Elias Andrade Gomes

ORCID: https://orcid.org/0000-0001-8083-9014 Universidade Federal do Ceará, Brasil E-mail: samuel.elias.a@gmail.com

Diana Valesca Carvalho

ORCID: https://orcid.org/0000-0001-5355-2309 Universidade Federal do Ceará, Brasil E-mail: dianacarvalho@ufc.br

Adriana Camurça Pontes Siqueira ORCID: https://orcid.org/0000-0002-2131-7598 Universidade Federal do Ceará, Brasil

E-mail: adriana.siqueira@ufc.br

Ana Erbênia Pereira Mendes

ORCID: https://orcid.org/0000-0001-7653-8956 Universidade Federal do Ceará, Brasil

E-mail: erbenia@ufc.br

\begin{abstract}
Resumo
O aumento de indivíduos com sobrepeso e obesidade no Brasil acentuou-se nas últimas décadas. As Doenças Crônicas Não Transmissíveis (DCNT’s), atualmente, são a principais causas de morte entre adultos. A alimentação balanceada, junto com outros fatores como a atividade física regular e o lazer, incidem positivamente na manutenção de peso e evitam o surgimento dessas doenças. Estudos sobre o consumo alimentar de universitários brasileiros observam grande incidência de inadequação alimentar, sobrepeso e obesidade entre os alunos. Considerando algumas características que auxiliam na garantia da qualidade de vida de estudantes universitários a partir da alimentação, o presente estudo teve como objetivo investigar as percepções nutricionais e as práticas alimentares de discentes do curso de gastronomia de uma universidade pública do Brasil. A pesquisa usou como método o estudo transversal, no qual buscou-se compreender a incidência de fatores relacionados ao contexto alimentar durante a vivência acadêmica. Os fatores observados foram perfil nutricional, consumo alimentar e conhecimento nutricional seguindo metodologias já validadas. Os sujeitos investigados foram discentes de ambos os sexos do curso de gastronomia do segundo ao oitavo semestre. Para coleta de dados, aplicou-se questionários on-line Google forms, com discentes que preenchiam os requisitos da investigação. Observou-se alta incidência de sobrepeso e obesidade, alto e médio conhecimento nutricional e inadequação em diferentes aspectos da alimentação na amostra. Os resultados, junto ao material bibliográfico levantado, revelaram que o conhecimento nutricional dos discentes, isoladamente, não se mostrou fator de mudança positiva nos hábitos alimentares e consequente manutenção de peso e prevenção de doenças.

Palavras-chave: Perfil nutricional; Consumo alimentar; Gastronomia.
\end{abstract}

\begin{abstract}
The number of overweight and obese individuals in Brazil has increased in recent decades. Chronic NonCommunicable Diseases (NCDs) are currently the leading cause of death among adults. Balanced diet, along with other factors such as frequent physical activity and leisure, positively affect weight maintenance and prevent the onset of these diseases. Studies on the food consumption of Brazilian university under graduates observe a high incidence of food inadequacy, overweight and obesity among students. Considering characteristics that help guarantee life quality from food among students, the present study aimed to investigate nutritional perceptions and eating practices of students in the gastronomy course at a public university in Brazil. The research used as a method the cross-sectional study, in which we sought to understand the incidence of factors related to the food context during the academic experience. The observed factors were nutritional profile, food consumption and nutritional knowledge following methodologies already validated. The investigated subjects were students of both gender in the gastronomy course from the second to the eighth semester. For data collection, Google forms online questionnaires were applied, with students who fulfilled the requirements of the investigation. The results indicate a high incidence of overweight and obesity, high and medium nutritional knowledge and inadequacy in different aspects of alimentation of the sample.
\end{abstract}


The results, together with the bibliographic material surveyed, revealed that the nutritional knowledge of the students, in isolation, was not a factor of positive change in eating habits and consequent weight maintenance and disease prevention.

Keywords: Nutricional profile; Food cosumption; Gastronomy.

\section{Resumen}

El aumento de personas con sobrepeso y obesidad en Brasil he aumentado en las últimas décadas. Las enfermedades crónicas no transmisibles (ENT) son actualmente la principal causa de muerte entre los adultos. La dieta equilibrada, junto con otros factores como la actividad física regular y el ocio, inciden positivamente en el mantenimiento del peso y previenen la aparición de estas enfermedades. Los estudios sobre el consumo de alimentos de estudiantes universitarios brasileños observan una alta incidencia de insuficiencia alimentaria, sobrepeso y obesidad entre los estudiantes. Considerando algunas características que ayudan a garantizar la calidad de vida de los estudiantes universitarios a partir de la alimentación, el presente estudio tuvo como objetivo investigar las percepciones nutricionales y prácticas alimentarias de los estudiantes del curso de gastronomía de una universidad pública de Brasil. La investigación utilizó como método el estudio transversal, en el que se buscó comprender la incidencia de factores relacionados con el contexto alimentario durante la experiencia académica. Los factores observados fueron perfil nutricional, consumo de alimentos y conocimiento nutricional siguiendo metodologías ya validadas. Los sujetos investigados fueron estudiantes de ambos sexos en el curso de gastronomía del segundo al octavo semestre. Para la recolección de datos se aplicaron cuestionarios en línea de formularios de Google, con estudiantes que cumplieron con los requisitos de la investigación. Hubo alta incidencia de sobrepeso y obesidad, conocimiento nutricional alto y medio e insuficiencia en diferentes aspectos de la alimentación de la muestra. Los resultados, junto con el material bibliográfico relevado, revelaron que el conocimiento nutricional de los estudiantes, de manera aislada, no fue un factor de cambio positivo en los hábitos alimentarios y consecuente mantenimiento de peso y prevención de enfermedades.

Palabras clave: Perfil nutricional; Consumo de comida; Gastronomía.

\section{Introdução}

O aumento de sobrepeso e obesidade incide a todas as faixas etárias da população brasileira, constituindo-se em fator de risco para Doenças Crônicas Não Transmissíveis (DCNT's). Seus sintomas aparecem de maneira lenta e gradativa e as complicações podem levar desde a incapacidade até a morte (BRASIL, 2014).

Essas doenças poderiam ser evitadas com um estilo de vida saudável, contemplando fatores como alimentação adequada, prática regular de exercícios físicos, lazer, controle de estresse e cuidados com si e com o meio. A promoção de uma alimentação saudável, em que haja o consumo de todos os nutrientes na dieta de maneira balanceada junto com o exercício físico resulta em importantes alterações na composição corporal e consequente diminuição das DCNT's (Barbosa et al., 2016).

A alimentação humana é investigada amplamente ao redor do mundo e a nutrição adequada é um dos requisitos básicos para a promoção da saúde e potencializa o desenvolvimento humano com qualidade de vida e cidadania. Ao se pensar na alimentação saudável como direito humano, atenta-se aos padrões alimentares adequados às necessidades biológicas e sociais compatíveis com sua faixa etária e fase da vida. Uma alimentação saudável deve assumir os significados socioculturais dos alimentos e aplicá-los em práticas alimentares conscientes (Brasil, 2012).

Para que se ocorra a adequação da alimentação faz-se necessário o aumento da compreensão dos indivíduos em todos os fenômenos relacionados à sua saúde para melhoria da qualidade de vida. O conhecimento das propriedades nutricionais dos alimentos, as porções diárias a serem ingeridas, a frequência de consumo alimentar e fatores relacionados mostram-se fundamentais para auxiliar na realização de mudanças positivas nos hábitos alimentares ao longo da vida dos indivíduos. Porém, fatores como baixa renda, exclusão social, baixa escolaridade, ou falta de informações disponíveis podem restringir o conhecimento dos indivíduos sobre alimentação saudável e favorecer o surgimento de doenças (Barbosa et al., 2016).

A transição para o meio acadêmico pode promover mudanças significativas no nível de atividade física e na qualidade da dieta de estudantes. Estudos recentes sobre o perfil nutricional e consumo alimentar de universitários brasileiros apresentam resultados preocupantes. Nota-se a grande prevalência de sobrepeso entre os estudantes e ingestão inadequada de alimentos marcadores de uma alimentação saudável como frutas, legumes e hortaliças (Marcondelli et al., 2008). 
Vários são os fatores que levam a essas mudanças alimentares e ao consequente reflexo na saúde dos estudantes após ingresso à universidade. Como exemplo citam-se as diversas atividades acadêmicas que influenciam nas escolhas dos alimentos e diminuem o tempo para realizar as refeições de forma adequada (Feitosa et al., 2010). Outros fatores como: independência, maturidade, raciocínio crítico e vida profissional também podem atuar como agentes nessas mudanças (de Souza Oliveira et al., 2017).

Os discentes do curso de gastronomia, em suas vivências acadêmicas, se deparam com novos insumos, texturas e sabores. Alguns fatores como a degustação de pratos em aulas práticas, a alta demanda de atividades acadêmicas e por vezes a inserção profissional em restaurantes e outros serviços de alimentação, podem refletir no consumo alimentar dessa população. Para estes, conhecer as propriedades nutricionais dos alimentos, além de proporcionar a realização de práticas alimentares individuais saudáveis e equilibradas, auxilia, também, na aplicação desses conhecimentos em suas vivências profissionais, assim como na pesquisa e no ensino acadêmico.

Ao se aprofundar nos aspectos nutricionais dos alimentos, aliado aos conhecimentos culturais, sociais, técnicos, biológicos e químicos, o gastrônomo é capaz de atuar ativamente como agente transformador de hábitos alimentares.

Este estudo justifica-se pela inexistência de pesquisas sobre práticas e percepções alimentares nos cursos de gastronomia, e por apresentar reflexões acerca da disseminação do conhecimento em alimentação saudável e da aplicação da educação alimentar e nutricional (EAN) para discentes de cursos que atuam nos setores da alimentação humana. A partir desse pressuposto, buscando a garantia da saúde e qualidade de vida dos estudantes, este estudo teve como objetivo investigar as percepções nutricionais e as práticas alimentares de discentes do curso de gastronomia da Universidade Federal do Ceará.

\section{Metodologia}

Trata-se de um estudo transversal que analisou as medidas antropométricas, consumo alimentar e conhecimento nutricional da população de discentes do curso de gastronomia da Universidade Federal do Ceará durante sua vivência acadêmica. Os critérios de inclusão estabelecidos foram alunos maiores de dezoito anos, com matrícula regular a partir do segundo semestre até o oitavo. O critério de exclusão foi estar cursando o primeiro semestre: isso se dá ao fato de os alunos desse período não estudarem nenhuma disciplina que aborde o tema pesquisado e ao pouco tempo de vivência na universidade.

A primeira etapa do estudo se caracterizou pelo quantitativo de discentes ativos no curso e quais preencheram os requisitos da pesquisa. Os instrumentos da pesquisa foram questões estruturadas aplicados virtualmente pelo pesquisador utilizando a plataforma Google Forms e divididas em quatro seções: Termo de Consentimento Livre e Esclarecido (TCLE), perfil alimentar, práticas alimentares e conhecimento nutricional. Os discentes foram convidados a responder os formulários via e-mail e redes sociais: WhatsApp e Instagram.

Para investigar as medidas antropométricas da população do estudo, foram incluídas duas questões abertas para que cada participante informasse seu peso e altura atuais. O perfil antropométrico foi identificado através do índice de massa corporal (IMC) que é a divisão do peso em quilogramas $(\mathrm{kg})$ pela altura em metros quadrados $\left(\mathrm{m}^{2}\right)$ de cada amostra. A partir do resultado, as amostras foram classificadas segundo os parâmetros da Organização Mundial da Saúde (OMS), que classifica para adultos: baixo peso (IMC $\left.\leq 18,5 \mathrm{~kg} / \mathrm{m}^{2}\right)$; peso adequado $\left(18,5<\mathrm{IMC}<25 \mathrm{~kg} / \mathrm{m}^{2}\right)$; sobrepeso $\left(25 \leq \mathrm{IMC}<30 \mathrm{~kg} / \mathrm{m}^{2}\right)$ e obesidade (IMC $\geq 30,00 \mathrm{~kg} / \mathrm{m}^{2}$ ) (OMS, 1995).

Para se avaliar as práticas alimentares dos discentes, utilizou-se os formulários de marcadores de consumo alimentar utilizado na rotina da atenção básica, elaborado pelo Ministério da Saúde (Brasil, 2015). Sua temática vai ao encontro das recomendações do Guia Alimentar para a População Brasileira. O questionário apresenta seis perguntas baseadas no consumo alimentar do dia anterior e duas perguntas referentes ao comportamento alimentar. 
Para identificar o conhecimento nutricional utilizou-se um questionário baseado na escala de conhecimento (Harnack et al., 1997), aplicada no National Health Interview Survey Cancer Epidemiologye traduzida, adaptada e validada (Scagliusi et al., 2006) contendo 2 questões abertas e 10 de múltipla escolha, sendo um ou mais itens corretos para cada questão. Cada item correto equivale a 1 ponto. Pontuações totais entre zero e seis, entre sete e dez, e acima de dez indicam, respectivamente, baixo, moderado e alto conhecimento nutricional.

\section{Resultados e Discussão}

Responderam ao questionário da pesquisa 42 estudantes, 24 do sexo masculino e 18 do sexo feminino. Esse número representou $24,7 \%$ do total de alunos com matrícula ativa a partir do segundo semestre no curso de gastronomia durante a realização desta pesquisa. A priori, o questionário seria aplicado durante o período letivo, o que acarretaria em resultados de perfil alimentar condizentes com a rotina acadêmica. Porém, devido à pandemia de COVID-19 e consequente suspensão das aulas, a aplicação ocorreu em um período em que os discentes se encontravam possivelmente em casa, o que pode refletir em seus hábitos alimentares domiciliares.

\subsection{Perfil Nutricional}

Embora a maior parte da amostra (52\%) tenha apresentado IMC de peso adequado, identificou-se elevado percentual de estudantes acima do peso, na classificação de sobrepeso (36\%) e obesidade (12\%) (Figura 1).

Figura 1 - Classificação do IMC da amostra de alunos do curso de gastronomia de uma universidade pública brasileira, 2020.

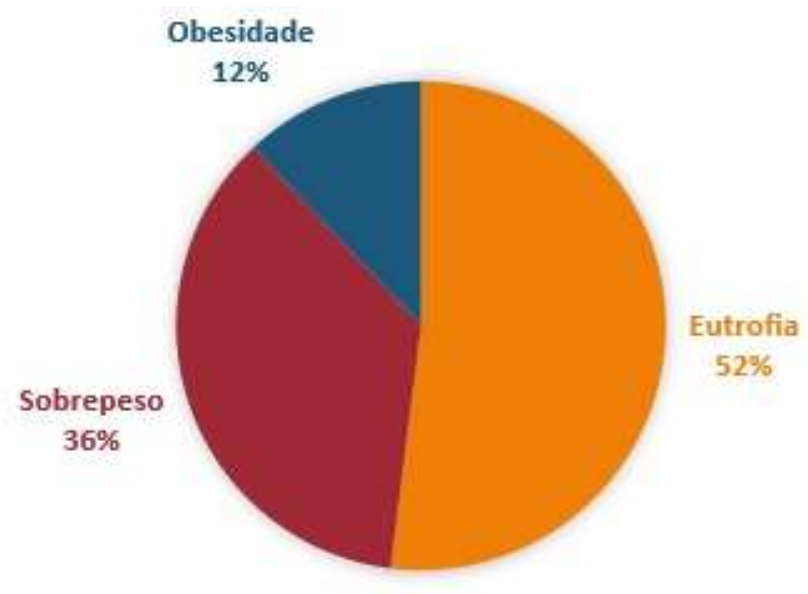

Fonte: Autores.

Os estudos sobre estado nutricional de universitários no Brasil apresentam índices de sobrepeso e obesidade muito variados, sendo comum o ganho de peso em ambos os sexos a partir da vida acadêmica. De Souza et al. (2017) pesquisaram o estado nutricional de estudantes de nutrição de uma universidade pública na cidade de Lagarto, no estado de Sergipe. O estado nutricional antropométrico dos estudantes apresentou $71 \%$ da amostra eutrófica, seguida de excesso de peso (sobrepeso e obesidade) com $22 \%$. Feitosa et al. (2010) pesquisando estado nutricional de universitários do nordeste do Brasil, observaram que a maioria da amostra se encontrava no estado eutrófico (69,6\%), enquanto 12,6\% encontravam-se com baixo peso, e 17,9\% apresentavam sobrepeso/obesidade. A pesquisa de Marcondelli et al. (2008) observou os seguintes resultados para estudantes universitários da área da saúde: $13,2 \%$ abaixo do peso, $75,4 \%$ com peso dentro do normal, 10,0\% com sobrepeso e 
1,4\% considerados obesos. Miranda et al. (2014) em trabalho com estudantes de cursos de Ciências Aplicadas da UNICAMP observaram $7 \%$ de sobrepeso e $4 \%$ de obesidade em mulheres, e $18 \%$ de sobrepeso e $6 \%$ de obesidade em homens.

Pesquisas com estudantes universitários em diferentes países também têm sido relatado. Lloyd-Richardson et al. (2009) observaram 904 alunos ingressantes em uma universidade pública dos Estados Unidos, evidenciando um ganho de peso médio de 3,5 $\mathrm{kg}$ em ambos os sexos a partir da vida acadêmica. Estudo envolvendo estudantes de medicina romenos da Universidade de Medicina e Farmácia "Iuliu Hatieganu", Cluj-Napoca verificou prevalência de excesso de peso em 30\% dos estudantes do sexo masculino, enquanto no sexo feminino a prevalência foi de $4,4 \%$ e associou o excesso de peso a baixo desempenho acadêmico, tabagismo e consumo excessivo de álcool (Brumboiu et al., 2018). Também, em estudo com universitários em Camarões, os resultados mostraram alta prevalência de má nutrição com base no IMC (baixo peso 4,9\%, sobrepeso $21,6 \%$ e obesidade 3,0\%) entre estudantes de medicina do segundo ano de três universidades estaduais (Bede et al., 2020).

Nota-se, portanto, que a amostra de estudantes pesquisada neste estudo apresentou índices mais elevados de sobrepeso e obesidade do que os observados entre outros estudantes universitários brasileiros e também de universitários de outros países, indicando que a cultura local pode ser um fator expressivo a se considerar sobre hábitos alimentares (Maciel, 2006).

\subsection{Consumo Alimentar}

O questionário alimentar coletou informações de comportamento, frequência e recordatório alimentar. A Figura 2 apresenta a porcentagem de adesão dos indivíduos à cada uma das refeições diárias.

Figura 2 - Adesão às refeições diárias da amostra de estudantes do curso de gastronomia de uma universidade pública brasileira, 2020.

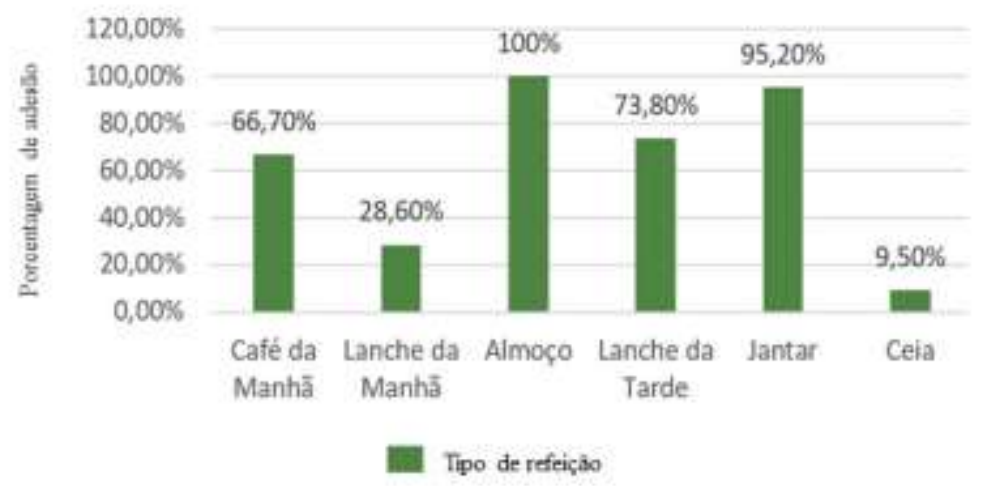

Fonte: Autores.

A refeição principal dos estudantes de gastronomia foi o almoço, seguida do jantar. O lanche da tarde $(73,8 \%)$ teve mais adesão que as refeições da manhã: café da manhã $(66,7 \%)$ e lanche da manhã $(28,6 \%)$. Isso demonstra que boa parcela desses estudantes $(33,3 \%)$ faz do almoço a primeira refeição do dia. Um estudo qualitativo com universitários apresentou diversos fatores que afetaram sua rotina alimentar. Com relação ao consumo do café da manhã, os autores citam: morarem sozinhos, falta de tempo e não terem se adaptado ao ritmo da universidade. Geralmente o café da manhã desses estudantes é realizado em casa e sozinho, mesmo daqueles que moram junto ao núcleo familiar. Os que não conseguem se alimentar em casa, geralmente comem lanches rápidos em estabelecimentos comerciais (Borges \& Lima Filho, 2004). 
Há semelhanças nos resultados do presente estudo com a avaliação de Busato et al. (2015) com estudantes que residem em moradia universitária, na qual 89\% considera o horário de almoço como refeição principal, o jantar foi substituído por refeições mais leves por mais da metade (62\%) dos estudantes e o desjejum também aparece como a refeição mais negligenciada.

A pesquisa de Alves e Boog (2007) também mostra resultados semelhantes: 72\% dos alunos realizavam o almoço completo (com alimentos à base de carboidratos, proteínas, vitaminas e vegetais), porém 63\% classificou o jantar como incompleto, cerca de 30\% afirmaram omitir o café da manhã e $20 \%$ afirmaram não realizar um desjejum completo (com alimentos reguladores ou com fonte de cálcio e carboidratos). Também, um estudo sobre comportamento alimentar de recém ingressos em uma universidade pública verificou que $60 \%$ dos estudantes não realizavam as três refeições principais (desjejum, almoço e jantar) e 37\% não realizava desjejum (Vieira et al., 2002).

Refeições irregulares e omissão de refeições também têm sido observado em estudos com universitários de outros países. Pular o café da manhã e comer entre as refeições foram comuns entre estudantes de uma universidade privada do sul da Nigéria (Omage \& Omuemu, 2018). Também, Bede et al. (2020) observaram que grande parte dos estudantes pesquisados negligenciavam o desjejum, sendo o jantar a refeição mais consumida e muitos relataram o consumo de lanches fora do contexto das três principais refeições. O motivo mais frequentemente referido pelos estudantes para pular uma refeição foi atribuído ao fato de estarem ocupados.

Com relação ao consumo alimentar, a Figura 3 apresenta informações sobre consumo de alguns alimentos referente ao dia anterior.

Figura 3 - Consumo alimentar da amostra de estudantes do curso de gastronomia de uma universidade pública brasileira, 2020.

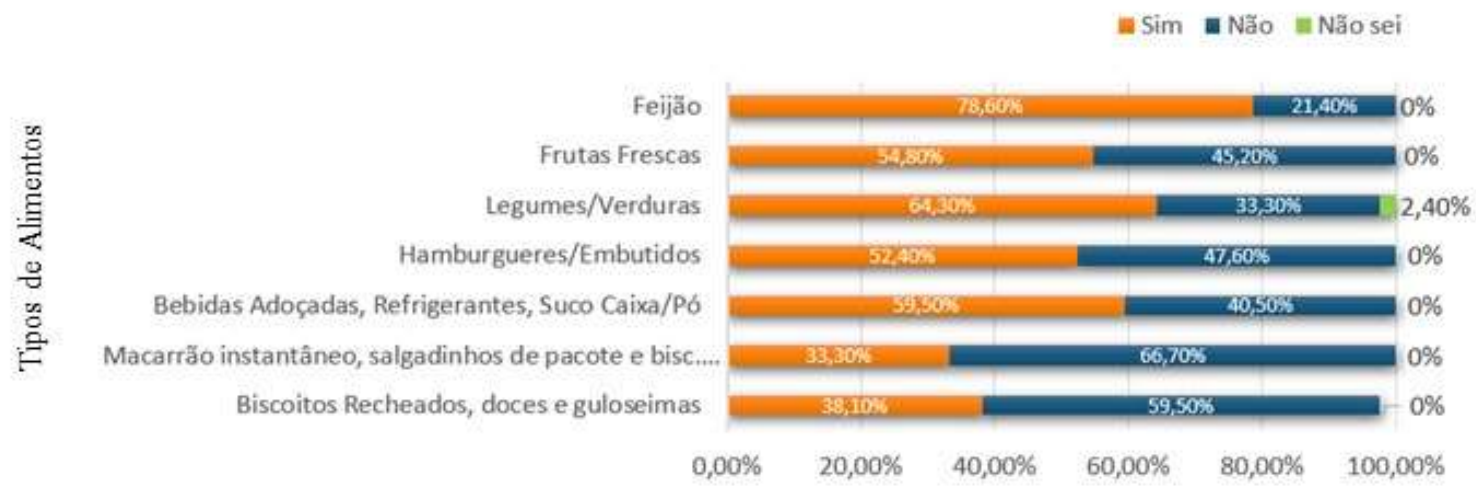

Porcentagem de consumo

Fonte: Autores.

Dos alimentos consumidos pelos universitários pesquisados, o feijão ainda representa um alimento de destaque, com consumo por parte de 78,6\% dos estudantes. Por possuírem alto teor de fibras e quantidade moderada de calorias por grama, o consumo das leguminosas, em nosso país melhor representado no feijão, confere alto poder de saciedade, evitando assim o consumo excessivo de calorias totais (Brasil, 2014). Os dados condizem com o estudo de Feitosa et al. (2010) em que 76\% dos estudantes entrevistados consumiam essa leguminosa mais de quatro vezes por semana, atingindo o padrão das recomendações diárias para esse grupo de alimentos. 
$\mathrm{Na}$ amostra estudada de universitários 33,3\% relataram não consumir legumes e verduras e 42,5\% frutas, o que pode significar a substituição desses alimentos por outros de menor importância nutricional. Estudo mostrou que o consumo semanal de verduras, legumes e frutas por estudantes universitários é baixo, apresentando inadequação de 84,4\% para legumes e verduras e 67,7\% para frutas, independente do gênero (Feitosa et al., 2010). Também, a pesquisa de Alves E Boog (2007) identificou que cerca de $48 \%$ dos acadêmicos não havia consumido nenhuma fruta no dia anterior e cerca de $22 \%$ afirmou nunca comprar hortaliças no mercado.

Corroborando ainda mais com esses resultados, uma pesquisa com estudantes de cursos da área de ciências aplicadas, verificou que a amostra estudada apresentava consumo deficiente de cereais, frutas e hortaliças entre alunos de todos os cursos estudados (Miranda et al., 2014).

Algumas pesquisas com estudantes universitários de outros países apresentam resultados conflitantes. No estudo de Bede et al. (2020), frutas eram consumidas regularmente por $26,1 \%$ dos alunos e $25,1 \%$ raramente ou nunca comiam frutas, enquanto isso, vegetais eram consumidos regularmente por $24,6 \%$, observando-se valor inferior ao consumo registrado pelos estudantes deste estudo. Por outro lado, Omage e Omuemu (2018) verificou consumo de frutas e vegetais por 51,5\% e 65\% dos estudantes pesquisados, respectivamente.

O consumo de frutas e verduras entre estudantes é menor que 400g/dia, o padrão recomendado pela OMS (Maciel, 2006). O consumo desses alimentos é reconhecido por ajudar na diminuição de incidência de diversas doenças crônicas. Entretanto, neste estudo, nota-se um consumo insuficiente de frutas, verduras e legumes por parte de universitários, conforme recomendações estabelecidas pela OMS. O consumo de frutas, hortaliças e cereais integrais está associado ao teor de fibras na alimentação. As fibras atuam no aumento da saciedade, redução de apetite e retardo da absorção de carboidratos após as refeições (Miranda et al., 2014).

Sobre o consumo de alimentos processados e ultraprocessados, nota-se um consumo expressivo. Os hambúrgueres/embutidos são consumidos por mais da metade $(52,4 \%)$ dos estudantes. As bebidas adoçadas, refrigerantes e sucos processados, por exemplo, foram consumidos por 59,5\% (figura 3). Isso exemplifica a substituição de alimentos naturais por outros que apresentam maior facilidade de acesso e praticidade de consumo. Semelhante a esta pesquisa, estudos mostram elevado consumo de doces, refrigerantes e fast food entre estudantes universitários (Maciel, 2006; Marcondelli et al., 2008).

Sobre o comportamento dos indivíduos durante as refeições, observou-se que 78,6\% dos entrevistados realizam as refeições utilizando telas digitais. O consumo de alimentos não saudáveis e o subsequente ganho de peso estão diretamente relacionados ao tempo despendido em frente à TV. Entre os motivos para que isso ocorra, cita-se a inatividade física e o consumo de alimentos hipercalóricos durante essa atividade (Oliveira et al., 2016). O Guia Alimentar para a População Brasileira (2014) recomenda a não utilização de televisores e telefones celulares durante as refeições. A alimentação feita em horários regulares, com a devida atenção e sem envolver outras atividades evita que se coma rapidamente e favorece ambientes mais adequados para se comer: com mesas, utensílios apropriados e em companhia (BRASIL, 2014).

No Brasil, as produções científicas sobre hábitos alimentares de estudantes universitários ainda estão em crescimento. Uma revisão do tema foi realizada por Loureiro (2016) que observou o crescimento do número de publicações nos últimos anos. De maneira geral as pesquisas com os estudantes evidenciam ingestão dietética de micronutrientes inadequada e baixo consumo de alimentos marcadores de uma alimentação saudável.

\subsection{Conhecimento Nutricional}

Dos indivíduos da pesquisa, $88,1 \%$ responderam que se interessam pelo tema nutrição e $19 \%$ já cursou ou está cursando a disciplina de Nutrição, ofertada semestralmente pelo curso de gastronomia da UFC. 
O questionário de conhecimento nutricional colheu dados referentes ao conhecimento atual em nutrição. Todos os participantes responderam corretamente que ao se consumir certos tipos de alimentos as chances de incidência das principais DCNT's diminuem. As doenças resultantes de uma má alimentação mais citadas pelos estudantes foram diabetes, seguidas de doenças cardíacas, obesidade e câncer (Figura 4), evidenciando o conhecimento dos estudantes com relação as doenças provenientes de uma má alimentação. É importante ressaltar que os estudantes citaram mais de uma doença, totalizando 114 respostas.

Figura 4 - Doenças mais citadas (em número) pela amostra de estudantes do curso de gastronomia de uma universidade pública brasileira, 2020.

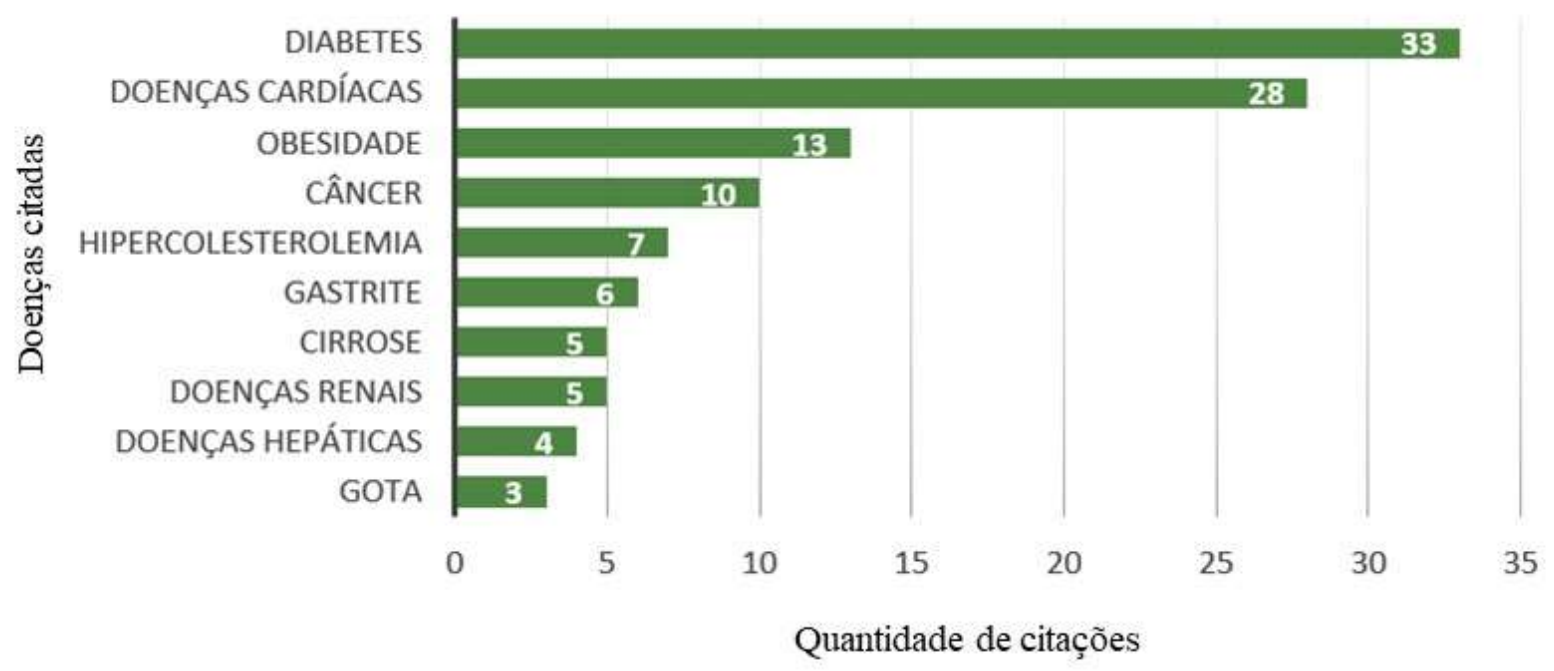

Fonte: Autores.

A grande maioria da amostra estudada considera que o câncer está relacionado com a alimentação (71,4\%), enquanto 28,6\% dizem que provavelmente esteja relacionado com a alimentação e apontam algumas atitudes que ajudam a diminuir a incidência de certos tipos de câncer como o consumo de frutas e hortaliças, mudança no consumo de alimentos e consumo de fibras (Figura 5). Porém, poucos reconhecem que o sedentarismo, o consumo de alimentos processados e Junk Food podem causar o câncer, pois apenas 2,4\% respondeu a cada uma das opções (Figura 5). Esses dados são preocupantes, pois observa-se grande consumo desses alimentos por parte dos estudantes. 
Figura 5 - Conhecimento da amostra de estudantes do curso de gastronomia de uma universidade pública brasileira sobre quais atitudes alimentares podem diminuir a incidência de alguns tipos de câncer, 2020.

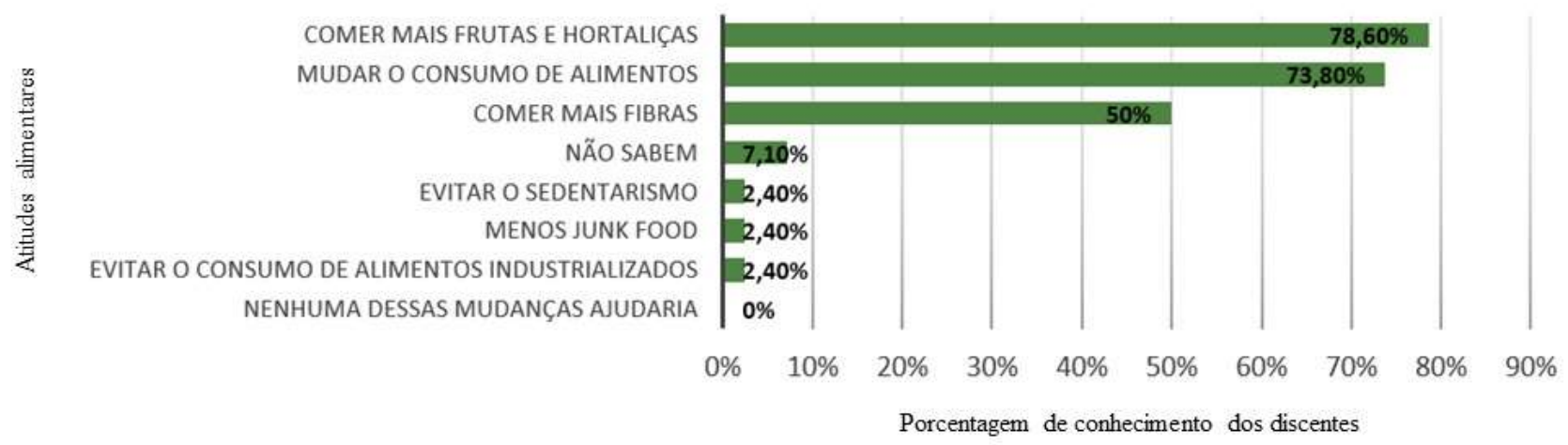

Fonte: Autores.

Todos os estudantes já tinham ouvido falar em fibras alimentares e a maioria identificou corretamente seu teor em alguns alimentos como o feijão e o farelo de trigo, porém a maioria não tinha conhecimento sobre o teor de fibras da cenoura: $71,4 \%$ da amostra não sabia que era mais rica em fibras que a alface (Figura 6). Os resultados sobre conhecimento de teor de lipídeos indicam média de $69 \%$ de acerto entre as três perguntas. Nota-se, porém que $31 \%$ não sabiam que um copo de leite possui mais gorduras que um copo de refrigerante e $28,6 \%$ não sabiam que um pedaço de bolo simples possui mais lipídeos que uma fatia de pão integral (Figura 7).

Figura 6 - Conhecimento da amostra de estudantes de gastronomia de uma universidade pública brasileira sobre o comparativo do teor de fibras entre alimentos, 2020.
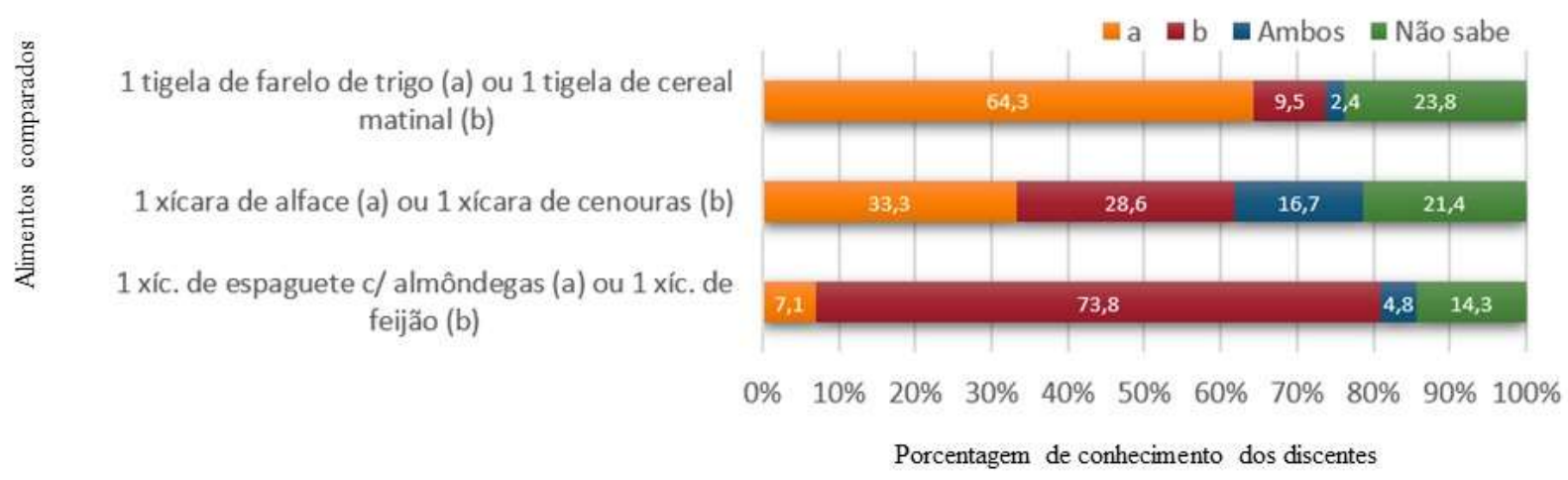

Fonte: Autores. 
Figura 7 - Conhecimento da amostra de estudantes de gastronomia de uma universidade pública brasileira sobre o comparativo do teor de lipídeos entre alimentos, 2020.

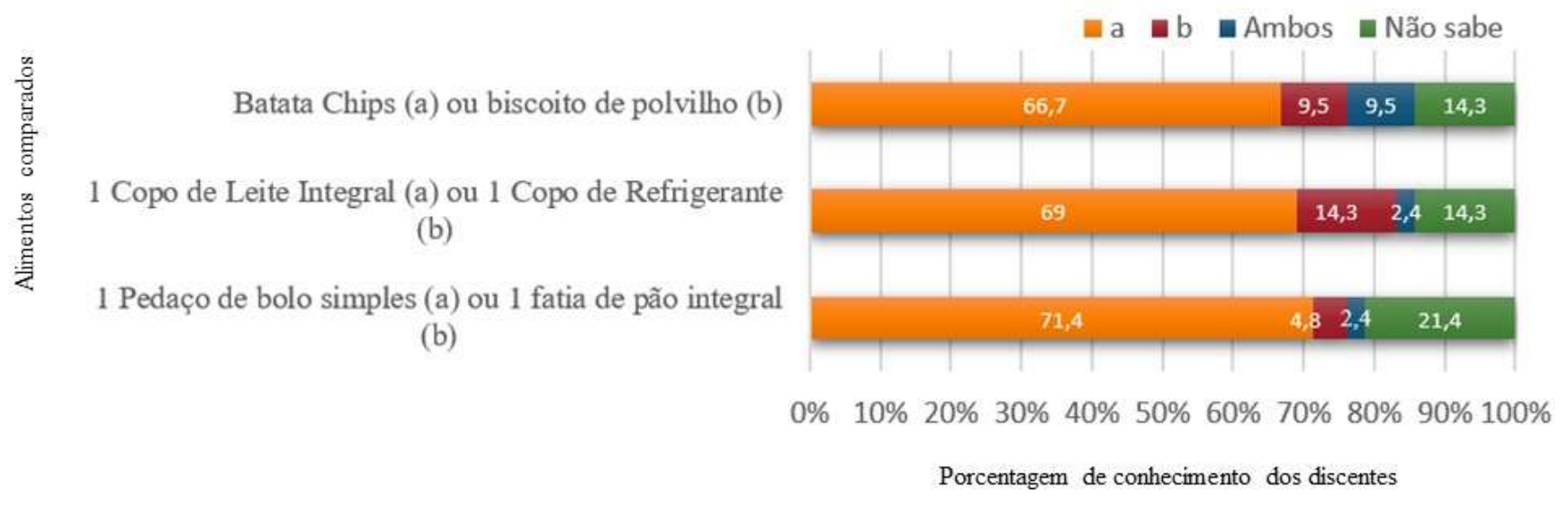

Fonte: Autores.

Na última questão, os participantes deveriam identificar quantas porções de frutas e hortaliças uma pessoa deveria consumir por dia para ter uma boa saúde. Uma síntese das respostas foi realizada, conforme a Figura 8.

Figura 8- Conhecimento da amostra de estudantes de gastronomia de uma universidade pública brasileira sobre consumo diário de frutas e hortaliças, 2020.

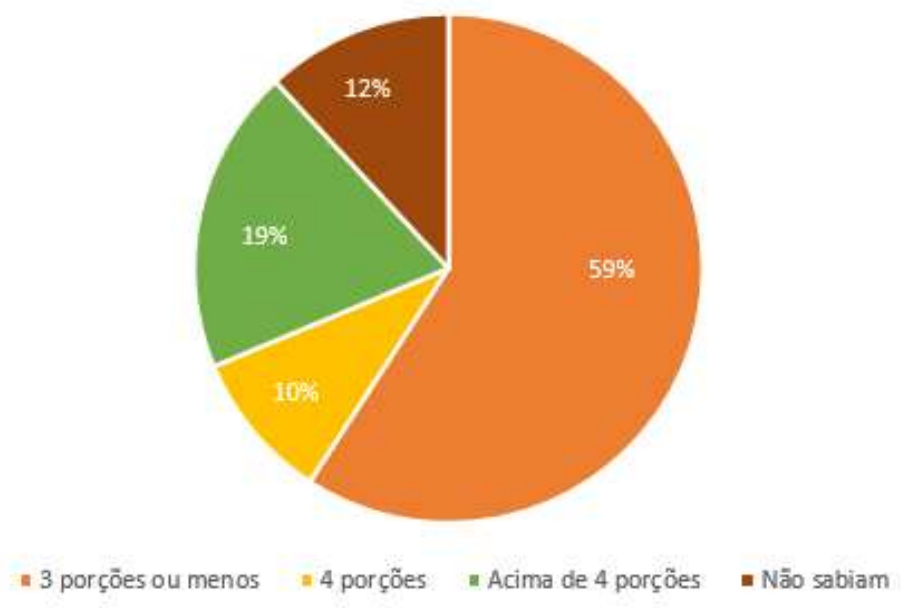

Fonte: Autores.

A maioria dos estudantes pesquisados desconhecem a recomendação do consumo de frutas e hortaliças, considerando que o consumo adequado seria de 3 ou menos porções/dia, enquanto a OMS recomenda 5 porções diárias, pelo menos cinco dias da semana, de frutas, verduras e hortaliças.

Ao analisar o conhecimento dos estudantes seguindo a metodologia de Scagliusi (2006), o resultado total indica que a maioria dos estudantes entrevistados (52\%) possui alto conhecimento nutricional, seguido de conhecimento moderado (43\%) e baixo conhecimento nutricional $(5 \%)$. Percebe-se com este resultado que o conhecimento nutricional dos estudantes não foi acompanhado por um perfil nutricional eutrófico. Os estudos mostram pouca influência do nível de conhecimento nutricional sob a composição corporal dos indivíduos, pois as práticas alimentares são determinadas por aspectos de cunho cultural, educacional e econômico, sendo o conhecimento nutricional importante, mas não um fator isolado para mudar o 
comportamento alimentar (Dattilo et al., 2009; Loureiro, 2016; Montero Bravo et al., 2006; O’brien \& Davies, 2007; Rêgo et al., 2015).

\section{Conclusão}

A partir dos dados analisados, destacam-se a alta incidência de discentes do curso de gastronomia da UFC com sobrepeso e obesidade na amostra, consumo inadequado de frutas e verduras, elevado consumo de alimentos ultraprocessados e frequência alimentar inadequada; porém, com alto/médio conhecimento nutricional.

O conhecimento nutricional pode apresentar relações com a seleção alimentar, porém não se mostra, isoladamente, fator de adesão de práticas alimentares saudáveis e consequente manutenção de peso e prevenção de doenças entre os estudantes observados, fato que estimula a discussão em estratégias de promoção de saúde mais diretas, que priorizem a incorporação do conhecimento adquirido em mudanças significativas nos hábitos e consumo alimentar dessa população.

Devido ao baixo número de pesquisas com essa população, não se tem estabelecido a real influência do espaço universitário em comportamentos relacionados à alimentação, manutenção de peso e prevenção de doenças entre os estudantes. Destaca-se a necessidade de mais estudos científicos para que se elucide a real influência dos conhecimentos em nutrição na adesão de práticas alimentares saudáveis.

\section{Referências}

Alves, H. J., \& Boog, M. A. F. (2007). Comportamento alimentar em moradia estudantil: um espaço para a promoção da saúde. Revista de Saúde Pública, 41 (2), 197-204.

Barbosa, L. B., Vasconcelos, S. M. L., Correia, L. O. dos S., \& Ferreira, R. C. (2016). Estudos de avaliação do conhecimento nutricional de adultos: Uma revisão sistemática. Ciência \& Saúde Coletiva, 21 (2), 449-462.

Bede, F., Cumber, S. N., Nkfusai, C. N., Venyuy, M. A., Ijang, Y. P., Wepngong, E. N., \& Kien, A. T. N. (2020). Dietary habits and nutritional status of medical school students: The case of three state universities in Cameroon. The Pan African Medical Journal, $35,15$.

Borges, C. M., \& Lima Filho, D. de O. (2004). Hábitos alimentares dos estudantes universitários: Um estudo qualitativo. Seminário em Administração, FEA$U S P$, agosto de.

Brasil. (2012). Marco de referência de educação alimentar e nutricional para as políticas públicas. Ministério do Desenvolvimento Social e Combate à Fome, Secretaria Nacional de Segurança Alimentar e Nutricional.

Brasil. (2014). Guia alimentar para a população brasileira (2a ed). Ministério da Saúde, Secretaria de Atenção à Saúde, Departamento de Atenção Básica.

Brasil. (2015). Orientações para avaliação de marcadores de consumo alimentar na atenção básica. Ministério da Saúde, Secretaria de Atenção à Saúde, Departamento de Atenção Básica. http://189.28.128.100/dab/docs/portaldab/publicacoes/marcadores_consumo_alimentar_atencao_basica.pdf

Brumboiu, M. I., Cazacu, I., Zunquin, G., Manole, F., Mogosan, C. I., Porrovecchio, A., Peze, T., Tavolacci, P., \& Ladner, J. (2018). Nutritional status and eating disorders among medical students from the Cluj-Napoca University centre. Clujul Medical, 91(4), 414-421.

Busato, M. A., Pedrolo, C., \& Gallina, L. S., Rosa, L. (2015). Ambiente e alimentação saudável: percepções e práticas de estudantes universitários. Semina: Ciências Biológicas e da Saúde, 36 (2), 75-84.

Dattilo, M., Furlanetto, P., Kuroda, A. P., Nicastro, H., Coimbra, P. C. F. C., \& Simony, R. F. (2009). Conhecimento nutricional e sua associação com o índice de massa corporal. Nutrire Rev. Soc. Bras. Aliment. Nutr, 34(1), 75-84.

de Souza Oliveira, J., de Oliveira Santos, D., Rodrigues, S. J. M., de Oliveira, C. C., \& da Costa Souza, A. L. (2017). Avaliação do perfil sociodemográfico, nutricional e alimentar de estudantes de nutrição de uma universidade pública em Lagarto-SE. Revista da Associação Brasileira de Nutrição-RASBRAN, 8 (2), $37-42$.

Feitosa, E. P. S., Dantas, C. A. de O., Andrade-Wartha, E. R. S., Marcellini, P. S., \& Mendes-Netto, R. S. (2010). Hábitos alimentares de estudantes de uma universidade pública no Nordeste, Brasil Food habits of students of one public university of Northeast, Brazil. Alimentos e Nutrição Araraquara, 21 (2), 225230.

Harnack, L., Block, G., Subar, A., Lane, S., \& Brand, R. (1997). Association of cancer prevention-related nutrition knowledge, beliefs, and attitudes to cancer prevention dietary behavior. Journal of the American Dietetic Association, 97 (9), 957-965.

Lloyd-Richardson, E. E., Bailey, S., Fava, J. L., \& Wing, R. (2009). A prospective study of weight gain during the college freshman and sophomore years. Preventive Medicine, 48 (3), 256-261. 
Research, Society and Development, v. 10, n. 3, e33110313275, 2021

(CC BY 4.0) | ISSN 2525-3409 | DOI: http://dx.doi.org/10.33448/rsd-v10i3.13275

Loureiro, M. P. (2016). Estado nutricional e hábitos alimentares de universitários. Segurança Alimentar e Nutricional, 23 (2), $955-972$.

Maciel, É. S. (2006). Qualidade de vida: análise da influência do consumo de alimentos e estilo de vida. Dissertação de Mestrado, Escola Superior de Agricultura Luiz de Queiroz, Universidade de São Paulo, Piracicaba.

Marcondelli, P., Costa, T. H. M. da, \& Schmitz, B. de A. S. (2008). Nível de atividade física e hábitos alimentares de universitários do $3^{\circ}$ ao $5^{\circ}$ semestres da área da saúde. Revista de Nutrição, 21 (1), 39-47.

Miranda, M. L. P., Kamiji, M. M., Torezzan, C., \& Antunes, A. E. C. (2014). Avaliação antropométrica e análise do consumo alimentar de universitários da Faculdade de Ciências Aplicadas-UNICAMP. Segurança Alimentar e Nutricional, 21 (2), 461-468.

Montero Bravo, A., Úbeda Martín, N., \& García González, A. (2006). Evaluación de los hábitos alimentarios de una población de estudiantes universitarios en relación con sus conocimientos nutricionales. Nutrición hospitalaria, 21 (4), 466-473.

O’brien, G., \& Davies, M. (2007). Nutrition knowledge and body mass index. Health education research, 22(4), 571-575.

Oliveira, J. S., Barufaldi, L. A., Abreu, G. de A., Leal, V. S., Brunken, G. S., Vasconcelos, S. M. L., Santos, M. M. dos, \& Bloch, K. V. (2016). ERICA: Uso de telas e consumo de refeições e petiscos por adolescentes brasileiros. Revista de Saúde Pública, 50, 7s.

Omage, K., \& Omuemu, V. O. (2018). Assessment of dietary pattern and nutritional status of undergraduate students in a private university in southern Nigeria. Food science \& nutrition, 6 (7), 1890-1897.

OMS, O. M. da S. (1995). Physical status: The use and interpretation of anthropometry. Geneva: WHO. https://www.who.int/childgrowth/publications/physical_status/en/

Rêgo, J. T. P. do, Silva, T. A. L. da, Medeiros, R. M. V., Barboza, R. R., Medeiros, J. A. de, Dantas, P. M. S., \& Miranda, H. F. de. (2015). Conocimiento nutricional y estado antropométrico de atletas universitarios. Revista Brasileira de Medicina do Esporte, 21 (6), 447-450.

Scagliusi, F. B., Polacow, V. O., Cordás, T. A., Coelho, D., Alvarenga, M., Philippi, S. T., \& Lancha Júnior, A. H. (2006). Tradução, adaptação e avaliação psicométrica da escala de conhecimento nutricional do National Health Interview Survey Cancer Epidemiology. Revista de Nutrição, 19 (4), $425-436$.

Vieira, V. C. R., Priore, S. E., Ribeiro, S. M. R., Franceschini, S. do C. C., \& Almeida, L. P. (2002). Perfil socioeconômico, nutricional e de saúde de adolescentes recém-ingressos em uma universidade pública brasileira. Revista de Nutrição, 15 (3), $273-282$. 